\title{
Frame covariant formalism for fermionic theories
}

\author{
Kieran Finn ${ }^{1, \mathrm{a}}{ }^{\mathbb{D}}$, Sotirios Karamitsos ${ }^{1,2, \mathrm{~b}}$, Apostolos Pilaftsis ${ }^{1, \mathrm{c}}$ \\ ${ }^{1}$ Department of Physics and Astronomy, University of Manchester, Manchester M13 9PL, UK \\ ${ }^{2}$ Dipartimento di Fisica, Universitá di Pisa, Pisa, Italy
}

Received: 7 April 2021 / Accepted: 21 June 2021 / Published online: 2 July 2021

(C) The Author(s) 2021

\begin{abstract}
We present a frame- and reparametrisationinvariant formalism for quantum field theories that include fermionic degrees of freedom. We achieve this using methods of field-space covariance and the Vilkovisky-DeWitt (VDW) effective action. We explicitly construct a field-space supermanifold on which the quantum fields act as coordinates. We show how to define field-space tensors on this supermanifold from the classical action that are covariant under field reparametrisations. We then employ these tensors to equip the field-space supermanifold with a metric, thus solving a long-standing problem concerning the proper definition of a metric for fermionic theories. With the metric thus defined, we use well-established field-space techniques to extend the VDW effective action and express any fermionic theory in a frame- and field-reparametrisation-invariant manner.
\end{abstract}

\section{Introduction}

The same theory of physics can often be written in many different ways. By choosing a different parametrisation of the underlying degrees of freedom, one can make the theory appear very different. However, although intermediate calculations may differ, the predictions of the theory should be the same regardless of which parametrisation is chosen. This idea is known as reparametrisation invariance.

Although one might expect reparametrisation invariance to be satisfied almost trivially, there are actually several theories of physics for which it is not obeyed, most notably Quantum Field Theories (QFTs). As was first noted by Vilkovisky $[1,2]$, the standard definition of the quantum effective action [3-6] for QFTs yields different results off-shell for different parametrisations of the same theory [7-10]. These

\footnotetext{
a e-mail: kieran.finn@manchester.ac.uk (corresponding author)

b e-mail: sotirios.karamitsos@df.unipi.it

c e-mail: apostolos.pilaftsis@manchester.ac.uk
}

differences persist even when calculations are performed perturbatively using Feynman diagrams [11].

There have been several attempts to rectify these issues and define a formalism in which reparametrisation invariance is made manifest. Most progress in this direction has been made using the technique of field-space covariance [1,2,12-20]. In such an approach, the fields are interpreted as coordinates on a manifold, known as the field-space manifold. In this way, field redefinitions can be interpreted as diffeomorphisms of the field space. With this identification, we can lean on the vast resources of differential geometry to construct theories for which reparametrisation invariance is made manifest. All we need is to work exclusively with field-space tensors and ensure that all field-space indices are fully contracted. This formalism led to the reparametrisation invariant VilkoviskyDeWitt (VDW) effective action $[1,2,13]$.

The method of field-space covariance has been very successful for scalar field theories and has also been applied to gravity $[1,2,11,21-29]$ and gauge theories [1,2,7,30-32]. However the formalism has had less success in theories with fermionic degrees of freedom. While there have been some attempts to construct the VDW effective action for fermionic theories, these have either made no attempt to calculate the metric of the field-space $[13,14]$ or used a definition specific to the model under consideration [33,34]. As of yet, there has been no method to systematically define the field-space manifold for fermionic theories and this has potentially prevented the use of the VDW formalism from becoming more widespread.

The utility of generalizing the formalism to such theories should be readily apparent. Fermions are an integral part of all realistic quantum field theories, including the Standard Model [35-37]. By constructing the field space for theories with fermionic degrees of freedom, we will complete the formalism and will therefore be able to describe all quantum field theories in a way that is manifestly reparametrisation invariant. The goal of this paper is therefore to explicitly 
construct a field space for fermionic theories and thereby extend the applicability of the VDW effective action.

There are two main distinctions between fermionic fields and bosonic fields that affect how we construct the field space. First is the fact that fermions anti-commute with each other [38-40]. This requires the introduction of new mathematics to describe them even at the classical level - namely the introduction of Grassmannian fields [41]. To include such anticommuting degrees of freedom in this formalism, we must generalise the field space to a supermanifold [42-49]. This is a manifold in which some of the coordinates are Grassmannian. We will discuss the implications of this in more detail in Sect. 3.

In line with the conventions of the literature on supermanifolds, we will be using the prefix 'super' in several contexts. For example, we will employ the superdeterminant, supertranspose and even a notion of supersymmetric (these terms will be defined in due course). We wish to emphasise that, despite their names, these terms have nothing to do with Supersymmetry (SUSY) as it is usually understood in particle physics [50-52]. The formalism derived in this paper is applicable to all theories regardless of their underlying symmetry and thus applies equally to both Supersymmetric and non-Supersymmetric theories. We shall distinguish between the two concepts by writing terms related to supermanifolds in lower case. In contrast, when referring to theories with a physical Grassmannian symmetry, we shall always use the term Supersymmetry with a capital $\mathrm{S}$ or the acronym SUSY.

The second novelty of fermionic fields is that their equations of motion are only first order. This is in contrast to the equations of motion for bosonic fields, which are of second order and, in the absence of potential terms, constitute the geodesic equation of the field space. This difference arises from the fact that only single derivatives of fermions appear in the Lagrangian. Because of this, a new definition of the field-space metric is required for such theories. In fact, if we simply kept the same definition as we had for scalar field theories [11], we would get a singular metric when fermionic degrees of freedom are included.

The paper is laid out as follows. We start in Sect. 2 by reviewing the construction of the field space and the VDW effective action for scalar field theories. In Sect. 3 we then review the properties of supermanifolds and highlight the implications of the Grassmannian nature of fermions. In Sect. 4 we define a supermanifold for QFTs that include fermionic degrees of freedom. In Sect. 5 we show how fieldspace tensors on this supermanifold can be extracted from the Lagrangian. In Sect. 6 we show how to equip the field space supermanifold with a metric. We discuss what properties such a metric should possess in order to be consistent with known results and show how a metric with the correct properties can be constructed explicitly from the Lagrangian.
For illustration, we discuss a counterexample of a candidate metric with bad properties in Appendix A. In Sect. 7 we then combine these ideas with the VDW effective action in order to construct a fully reparametrisation invariant expression for the effective action. We then show some explicit examples of our construction in Sect. 8, before summarising our findings in Sect. 9.

\section{Field-space covariance for scalar fields}

The field-space covariant formalism was pioneered by Vilkovisky [1,2] and DeWitt [13]. In this section we review their construction of the field space for scalar field theories. This will help us identify what aspects of the construction must be altered when considering fermionic degrees of freedom.

We consider a theory of $N$ scalar fields $\phi^{A}$, collectively denoted as $\phi$ living in a fixed spacetime with metric $g_{\mu \nu}$. Such a theory is generally described by the Lagrangian

$\mathrm{L}=\frac{1}{2} k_{A B}(\boldsymbol{\phi}) g^{\mu \nu} \partial_{\mu} \phi^{A} \partial_{\nu} \phi^{B}-V(\boldsymbol{\phi})$,

where $k_{A B}(\phi)$ is a general model function for the kinetic term and $V(\phi)$ represents the scalar potential. We define the field space to be an $N$-dimensional manifold with coordinates $\phi^{A}$. In so doing, reparametrisations of the fields

$\phi^{A} \rightarrow \widetilde{\phi}^{A}(\phi)$

are interpreted as diffeomorphisms of the field space. We can then impose reparametrisation invariance using well-known techniques from differential geometry.

In order to take full advantage of these techniques, we equip the field-space manifold with a metric

$$
\begin{aligned}
G_{A B}= & \frac{g_{\mu \nu}}{4} \frac{\partial^{2} \mathrm{~L}}{\partial\left(\partial_{\mu} \phi^{A}\right) \partial\left(\partial_{\nu} \phi^{B}\right)}-\frac{\partial^{2} \mathrm{~L}}{\partial \phi^{A} \partial\left(\square \phi^{B}\right)} \\
& -\frac{\partial^{2} \mathrm{~L}}{\partial \phi^{B} \partial\left(\square \phi^{A}\right)},
\end{aligned}
$$

which, for the Lagrangian (2.1), gives $G_{A B}=k_{A B}$. Note that this definition differs from the one in [11] by the addition of the last two terms. These terms ensure that the metric does not depend on total derivatives appearing in the Lagrangian. The two definitions are identical for Lagrangians that contain no second derivative terms, as it is the case for (2.1).

With the metric defined in (2.3), we may introduce a connection into the field space via the Christoffell symbols

$$
\Gamma_{B C}^{A} \equiv \frac{1}{2} G^{A D}\left[\frac{\partial G_{B D}}{\partial \phi^{C}}+\frac{\partial G_{D C}}{\partial \phi^{B}}-\frac{\partial G_{B C}}{\partial \phi^{D}}\right],
$$


where $G^{A B}$ is the inverse of $G_{A B}$. Hence, we are able to define a covariant derivative on the field space:

$$
\begin{aligned}
& \nabla_{C} X^{A}=\frac{\partial X^{A}}{\partial \phi^{C}}+\Gamma_{C D}^{A} X^{D} \\
& \nabla_{C} X_{A}=\frac{\partial X_{A}}{\partial \phi^{C}}-\Gamma_{C A}^{D} X_{D}
\end{aligned}
$$

with obvious generalisation to higher order tensors.

To take account of the spacetime dependence of the fields, the field-space manifold is often generalised to the infinitedimensional configuration space manifold by taking each spacetime configuration of the fields as a different dimension on the manifold. This manifold can be described by coordinates

$\phi^{\widehat{A}} \equiv \phi^{A}\left(\boldsymbol{x}_{A}\right)$.

Here we have introduced a condensed notation in which an index with a hat represents both a discrete field-space index and a point in spacetime.

The metric of the configuration space is given by

$G_{\widehat{A} \widehat{B}}=\frac{g_{\mu \nu}}{4} \frac{\delta^{2} S}{\delta\left(\partial_{\mu} \phi^{\widehat{A}}\right) \delta\left(\partial_{\nu} \phi^{\widehat{B}}\right)}=G_{A B} \delta^{(4)}\left(\boldsymbol{x}_{A}-\boldsymbol{x}_{B}\right)$,

where $S=\int d^{4} x \sqrt{-g} \mathrm{~L}$ is the action and $\delta^{(4)}(x)$ is the covariant four-dimensional Dirac delta function, normalised such that

$\int \sqrt{-g} \delta^{(4)}(\boldsymbol{x}) d^{4} x=1$.

The definition of the configuration space metric leads to the following definition for the configuration space connections

$$
\begin{aligned}
\Gamma_{\widehat{B} \widehat{C}}^{\widehat{A}} & \equiv \frac{1}{2} G^{\widehat{A} \widehat{D}}\left[\frac{\delta G_{\widehat{B} \widehat{D}}}{\delta \phi^{\widehat{C}}}+\frac{\delta G_{\widehat{D} \widehat{C}}}{\delta \phi^{\widehat{B}}}-\frac{\delta G_{\widehat{B} \widehat{C}}}{\delta \phi^{\widehat{D}}}\right] \\
& =\Gamma_{B C}^{A} \delta^{(4)}\left(\boldsymbol{x}_{A}-\boldsymbol{x}_{B}\right) \delta^{(4)}\left(\boldsymbol{x}_{A}-\boldsymbol{x}_{C}\right)
\end{aligned}
$$

and hence configuration space covariant derivatives

$$
\begin{aligned}
& \nabla_{\widehat{C}} X^{\widehat{A}}=\frac{\delta X^{\widehat{A}}}{\delta \phi^{\widehat{C}}}+\Gamma_{\widehat{C} \widehat{D}}^{\widehat{A}} X^{\widehat{D}}, \\
& \nabla_{\widehat{C}} X_{\widehat{A}}=\frac{\delta X_{\widehat{A}}}{\delta \phi^{\widehat{C}}}-\Gamma_{\widehat{C} \widehat{A}}^{\widehat{D}} X_{\widehat{D}},
\end{aligned}
$$

with straightforward generalisation to higher order tensors. Here we have adopted the condensed Einstein-DeWitt notation [21] in which repeated configuration space indices imply summation over the discrete index and integration over spacetime.

Having set up the field space and configuration space technology, we may now use the results of Vilkovisky [1,2] and
DeWitt [13] to write a reparametrisation-invariant expression for the quantum effective action

$$
\begin{aligned}
\exp \left(\frac{i}{\hbar} \Gamma[\boldsymbol{\varphi}]\right)= & \int[\mathrm{D} \boldsymbol{\phi}] \sqrt{\operatorname{det} G(\boldsymbol{\phi})} \exp \left[\frac{i}{\hbar}(S[\boldsymbol{\phi}]\right. \\
& \left.\left.+\int d^{4} x \sqrt{-g} \frac{\partial \Gamma[\boldsymbol{\varphi}]}{\partial \varphi^{A}} \Sigma^{A}[\boldsymbol{\varphi}, \boldsymbol{\phi}]\right)\right]
\end{aligned}
$$

Here $\varphi$ stands for the mean field and

$$
\Sigma^{A}[\boldsymbol{\varphi}, \boldsymbol{\phi}]=\left(C^{-1}[\varphi]\right)_{B}^{A} \sigma^{B}[\boldsymbol{\varphi}, \boldsymbol{\phi}]
$$

is a linear combination of the tangent vectors to the geodesics connecting $\varphi$ and $\phi$, which are denoted $\sigma^{A}[\varphi, \phi]$. The matrix $C[\varphi]_{B}^{A}$ is chosen such that that $\left\langle\Sigma^{A}\right\rangle=0$ and thus all tadpole diagrams evaluate to zero. It can be shown that $C[\varphi]_{B}^{A}$ can be expanded as [13]

$$
\begin{aligned}
C_{B}^{A}[\boldsymbol{\varphi}] & =\left\langle\nabla_{B} \sigma^{A}[\boldsymbol{\varphi}, \boldsymbol{\phi}]\right\rangle \\
& =\left\langle\delta_{B}^{A}-\frac{1}{3} R_{C B D}^{A}[\varphi] \sigma^{C}[\boldsymbol{\varphi}, \boldsymbol{\phi}] \sigma^{D}[\boldsymbol{\varphi}, \boldsymbol{\phi}]+\cdots\right\rangle
\end{aligned}
$$

where $R_{C B D}^{A}$ is the field-space Riemann tensor and all covariant derivatives are taken with respect to the mean field $\boldsymbol{\varphi}$. Similarly, $\sigma^{A}[\boldsymbol{\varphi}, \boldsymbol{\phi}]$ can be expanded as $[1,2]$

$$
\begin{aligned}
-\sigma^{A}[\boldsymbol{\varphi}, \boldsymbol{\phi}]= & -\left(\varphi^{A}-\phi^{A}\right) \\
& +\frac{1}{2} \Gamma_{B C}^{A}[\varphi]\left(\varphi^{B}-\phi^{B}\right)\left(\varphi^{C}-\phi^{C}\right)+\cdots .
\end{aligned}
$$

The VDW effective action can be expanded perturbatively using the background field method to give, at one and two loop orders [15],

$$
\begin{aligned}
\Gamma^{(1)}[\varphi]= & -\frac{i}{2} \ln \operatorname{det} \nabla^{\widehat{A}} \nabla_{\widehat{B}} S, \\
\Gamma^{(2)}[\varphi]= & \frac{1}{8} \Delta^{\widehat{A B}} \Delta^{\widehat{C} \widehat{D}} \nabla_{(\widehat{A}} \nabla_{\widehat{B}} \nabla_{\widehat{C}} \nabla_{\widehat{D})} S \\
& -\frac{1}{12} \Delta^{\widehat{A} \widehat{B}} \Delta^{\widehat{C} \widehat{D}} \Delta \widehat{E}_{\widehat{E}} \\
& \times\left(\nabla_{(\widehat{A}} \nabla_{\widehat{C}} \nabla_{\widehat{E})} S\right)\left(\nabla_{(\widehat{B}} \nabla_{\widehat{D}} \nabla_{\widehat{F})} S\right),
\end{aligned}
$$

respectively, where $S=S[\varphi]$ is the action expressed in terms of the mean field $\varphi, \Delta^{\widehat{A} \widehat{B}}=\left(\nabla_{\widehat{A}} \nabla_{\widehat{B}} S\right)^{-1}$ is the covariant propagator, and the parentheses (...) denote symmetrisation with respect to the indices enclosed.

In addition to computing quantum corrections using the effective action formalism, we can also calculate corrections perturbatively using Feynman diagrams [53]. In order 
to maintain reparametrisation invariance, these should be calculated covariantly using covariant Feynman rules

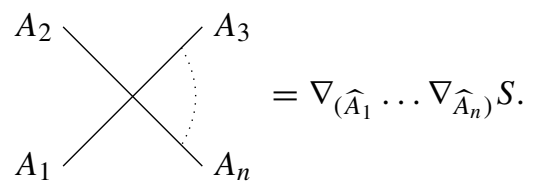

Notice that for theories with a non-trivial field space, these covariant Feynman rules differ from the usual ones. This is entirely by design, since the usual Feynman rules are not field-space tensors and so can lead to results that depend on the pararametrisation of the fields [11].

\section{Supermanifolds}

In order to construct a field-space manifold for fermionic theories we must extend the notion of a Riemannian manifold to include anticommuting coordinates. This is the definition of supermanifold [42-47]. In this section we review the basic properties of supermanifolds that will affect our construction of the field space. We encourage interested readers to consult $[48,49]$ for further details of the rich mathematics of this topic.

Originally, supermanifolds were invented in the context of Supersymmetry (SUSY) [54,55]. In this context, the usual spacetime is augmented with new Grassmannian coordinates and diffeomorphisms of the new superspace result in Grassmannian Noether symmetries. However, since their invention, the mathematics of supermanifolds has been developed as a subject in its own right and now has applications far beyond SUSY [56-58].

It is in this latter context that we employ supermanifolds in this paper. We are extending not the spacetime manifold, but the field-space manifold. Thus, the new Grassmannian diffeomorphisms are not physical Grassmannian symmetries of the theory, but merely reparametrisations of the fermionic fields. As with all reparametrisations, these field-space diffeomorphisms cannot be considered a symmetry in the traditional sense and there will, in general, be no Noether current or gauge degrees of freedom associated with them.

To set our notation, we consider a supermanifold with $n$ commutative coordinates and $m$ anti-commutative coordinates. We denote the coordinates $x^{\alpha}$ with $\alpha=(1,2 \ldots m+n)$. When we need to refer to the commutative and anticommutative coordinates separately we shall use $x^{A}$ for the former and $x^{I}$ for the latter, with letters from the start of the Latin alphabet indicating commutative coordinates and letters from the middle of the same alphabet indicating anticommutative coordinates.

The first subtlety we must consider is that when differentiating with respect to an anticommuting coordinate, we must specify whether we are differentiating from the left or from the right. The two types of differentiation are related by

$$
\vec{\partial}_{\alpha} X=(-1)^{\alpha(X+1)} X \overleftarrow{\partial_{\alpha}}
$$

In the above, we have introduced a new notation common throughout the literature on supermanifolds. The expressions in an exponent of -1 are not meant to be taken literally, but as labels standing for the grading of their respective quantities: 1 for anticommuting quantities and 0 for commuting quantities. Thus, the quantity $\alpha$ that appears in the prefactor on the RHS of (3.1) is not to be considered an index and is not summed over as would be expected by the Einstein summation convention. Instead, it should be regarded as a label that is 1 when $\alpha$ refers to an anticommuting coordinate and 0 when $\alpha$ refers to a commuting coordinate. Similarly, the $X$ in the exponent of -1 in (3.1) is to be considered a label that is 1 when $X$ is an anticommuting object and 0 when $X$ is a commuting object. Thus, (3.1) tells us that there is a factor of -1 between a left and right derivative, when differentiating a commuting object with respect to an anticommuting coordinate, but that they are identical in all other cases.

When performing a diffeomorphism

$x^{\alpha} \rightarrow \tilde{x}^{\alpha}=\tilde{x}^{\alpha}(\boldsymbol{x})$,

this difference between left and right derivatives leads to a distinction between left and right Jacobians. These are, respectively,

${ }_{\alpha} J^{\beta}=\frac{\vec{\partial}}{\partial x^{\alpha}} \tilde{x}^{\beta}, \quad{ }^{\beta} J_{\alpha}^{\mathrm{sT}}=\tilde{x}^{\beta} \frac{\overleftarrow{\partial}}{\partial x^{\alpha}}$

We distinguish between tensors that transform with a left or right Jacobian by writing the appropriate index to the right or left, respectively, of the tensor. Thus, $V^{\alpha}$ is a vector that transforms with a left Jacobian and ${ }^{\alpha} V$ is a vector that transforms with a right Jacobian, e.g. $\widetilde{V}^{\alpha}=V^{\beta}{ }_{\beta} J^{\alpha}$ and ${ }^{\alpha} \widetilde{V}={ }^{\alpha} J_{\beta}^{\text {sT }} \beta V$. Similarly, we define left and right covectors $V_{\alpha}$ and ${ }_{\alpha} V$ that transform with the left and right inverse Jacobians, respectively.

The superscript sT denotes the operation of supertransposition and is defined as

$$
\begin{aligned}
{ }^{\alpha} M_{\beta}^{\mathrm{sT}} & =(-1)^{\beta(\alpha+1)}{ }_{\beta} M^{\alpha}, \\
{ }_{\alpha} M_{\beta}^{\mathrm{sT}} & =(-1)^{\alpha+\beta+\alpha \beta}{ }_{\beta} M_{\alpha}, \\
{ }^{\alpha} M^{\beta \mathrm{sT}} & =(-1)^{\alpha \beta \beta} M^{\alpha} .
\end{aligned}
$$

Note that the rules of supertransposition are different depending on the index placement. The supertranspose satisfies the identities one would expect, namely 


$$
\begin{aligned}
\left(M^{\mathrm{sT}}\right)^{\mathrm{sT}} & =M, \\
\left(M^{-1}\right)^{\mathrm{sT}} & =\left(M^{\mathrm{sT}}\right)^{-1}, \\
(M N)^{\mathrm{sT}} & =N^{\mathrm{sT}} M^{\mathrm{sT}} .
\end{aligned}
$$

Note that this is in contrast to the regular transpose for which $(M N)^{\top} \neq N^{\top} M^{\top}$ in the presence of anticommuting coordinates.

The definition of the supertranspose leads to the notion of supersymmetric and anti-supersymmetric matrices, which satisfy $M^{\text {sT }}=M$ and $M^{\text {sT }}=-M$, respectively. Again, we emphasise that the definition of supersymmetric here should not be confused with the theory of SUSY.

Finally, we consider the superdeterminant, which is sometimes known as the Berezinian [43]. To define the superdeterminant, we consider a square rank-2 tensor on the supermanifold (sometimes known as a supermatrix), which has the form

${ }_{\alpha} M_{\beta}=\left(\begin{array}{cc}{ }_{A} A_{B} & { }_{A} C_{J} \\ { }_{I} D_{B} & { }_{I} B_{J}\end{array}\right)$.

Here ${ }_{A} A_{B}$ and ${ }_{I} B_{J}$ are $n \times n$ and $m \times m$ matrices of commuting numbers, respectively and ${ }_{A} C_{J}$ and ${ }_{I} D_{B}$ are $n \times m$ and $m \times n$ matrices of anticommuting numbers, respectively. The superdeterminant of such a matrix is given by

$$
\text { sdet } M=\frac{\operatorname{det}\left(A-C B^{-1} D\right)}{\operatorname{det} B} \text {. }
$$

The superdeterminant, defined in this way, is such that the Berezinian integral measure

$\sqrt{\operatorname{sdet}(M)} d^{n+m} x$

is invariant under diffeomorphisms of the supermanifold (3.2) [43]. This is true for any rank-2 tensor ${ }_{\alpha} M_{\beta}$.

\section{The field space for scalar-fermion theories}

In this section we construct the field space for a theory with $N$ real scalar fields and $M$ Dirac fermions. ${ }^{1}$ Recalling that, in 4dimensional spacetime, each Dirac fermion propagates four complex, or eight real, anticommuting degrees of freedom [38], we see that the field space for such a theory should be a supermanifold with $N$ commuting coordinates and $8 M$ anticommuting coordinates. Throughout this paper, we shall use the following set of coordinates to describe this fieldspace supermanifold

$\Phi^{\alpha}=\left(\phi^{A}, \psi_{a}^{1}, \bar{\psi}_{\dot{a}}^{1}, \psi_{a}^{2}, \bar{\psi}_{\dot{a}}^{2}, \ldots\right)$,

where the subscripts $a$ and $\dot{a}$ refer to the spinor components of the Dirac fermions. As done previously, we use

\footnotetext{
${ }^{1}$ A generalisation of this approach to theories with Weyl fermions will be straightforward.
}

Greek indices from the beginning of the alphabet, which run $1 \leq \alpha \leq N+8 M$, for the full supermanifold and, when we need to refer to them separately, we will use capital Latin letters from the beginning of the alphabet for the commuting coordinates and capital Latin letters from the middle of the alphabet for anticommuting coordinates. These run $1 \leq A \leq N$ and $1 \leq I \leq 8 M$, respectively. We will refer to all the field-space coordinates collectively as $\boldsymbol{\Phi}$.

A general field reparametrisation of the form

$$
\Phi^{\alpha} \rightarrow \widetilde{\Phi}^{\alpha}=\widetilde{\Phi}^{\alpha}(\boldsymbol{\Phi})
$$

is equivalent to a diffeomorphism of the field-space supermanifold and we can therefore enforce reparametrisation invariance using the techniques of differential supergeometry discussed in Sect. 3. We note that the transformation (4.2) is quite general and can, for instance, involve Grassmannian parameters. However, the transformation cannot depend on derivatives of the fields and so there are still certain transformations that are not captured by (4.2), for example the transformations of SUSY [50,51]. We believe extending the formalism to include such transformations will not be too difficult, but choose to leave such considerations for future work.

With the field space thus defined, let us write the most general Lagrangian for this theory using field-space tensors. Including terms up to quadratic order in derivatives, we get

$\mathrm{L}=\frac{1}{2} g^{\mu \nu} \partial_{\mu} \Phi_{\alpha}^{\alpha} k_{\beta}(\boldsymbol{\Phi}) \partial_{\nu} \Phi^{\beta}+\frac{i}{2} \zeta_{\alpha}^{\mu}(\boldsymbol{\Phi}) \partial_{\mu} \Phi^{\alpha}-U(\boldsymbol{\Phi})$.

This expression contains three model functions that define our theory: ${ }_{\alpha} k_{\beta}(\boldsymbol{\Phi})$ is a rank-2 field-space tensor, $\zeta_{\alpha}^{\mu}(\boldsymbol{\Phi})$ is a field-space covector and a spacetime vector and $U(\boldsymbol{\Phi})$ is a field-space and spacetime scalar. These model functions can, in general, depend on both the scalar and fermion fields in the theory, but not on their derivatives.

Let us analyse these three model functions in turn. The tensor ${ }_{\alpha} k_{\beta}$ is the scalar field-space metric. In the absence of fermion fields, this object just reduces to the metric (2.3). Because fermionic fields only enter the Lagrangian with a single derivative, we see that this tensor only has support in the bosonic sector, implying that

${ }_{\alpha} k_{I}={ }_{I} k_{\alpha}=0$.

Because of this, the tensor ${ }_{\alpha} k_{\beta}$ is singular and cannot play the role of the field-space metric in the presence of fermions.

Next we look at the potential term $U(\boldsymbol{\Phi})$. This term contains the scalar potential $V(\boldsymbol{\phi})$, as well as both the fermion mass terms and any momentum-independent interactions between the scalars and fermions such as Yukawa interactions [59]. As in the scalar case, the potential $U(\boldsymbol{\Phi})$ plays no role in the construction of the field-space manifold and so acts only as an external force. 
Finally, we consider the model function $\zeta_{\alpha}^{\mu}$. This model function has no analogue in a pure scalar field theory, because scalars cannot appear in the Lagrangian with a single derivative in a diffeomorphism invariant way. Since $\zeta_{\alpha}^{\mu}$ cannot depend on derivatives of the fields and there are no spacetime vectors in this theory, the spacetime index $\mu$ of this tensor can only come from a $\gamma^{\mu}$ matrix. This tells us why such a term cannot appear in a pure scalar theory, since there would then be no fermions to contract with the spinor indices of $\gamma^{\mu}$.

As an example to show the ubiquity of the expression (4.3), we consider a theory of free scalars and fermions with Lagrangian

$$
\begin{aligned}
\mathrm{L}= & \sum_{A \in \text { scalars }}\left[\frac{1}{2} g^{\mu \nu} \partial_{\mu} \phi^{A} \partial_{\nu} \phi^{A}-\frac{1}{2} m_{A}^{2}\left(\phi^{A}\right)^{2}\right] \\
& +\sum_{X \in \text { fermions }}\left[\frac{i}{2}\left(\bar{\psi}^{X} \gamma^{\mu} \partial_{\mu} \psi^{X}-\partial_{\mu} \bar{\psi}^{X} \gamma^{\mu} \psi^{X}\right)\right. \\
& \left.-m_{X} \bar{\psi}_{X} \psi_{X}\right] .
\end{aligned}
$$

Such a theory has the following model functions:

$$
\begin{aligned}
& { }_{\alpha} k_{\beta}=\left(\begin{array}{cc}
\delta_{A B} & \mathbf{0}_{N \times 8 M} \\
\mathbf{0}_{8 M \times N} & \mathbf{0}_{8 M \times 8 M}
\end{array}\right), \\
& \zeta_{\alpha}^{\mu}=\left(\mathbf{0}_{N}, \bar{\psi}_{\dot{a}}^{1} \gamma_{\dot{a} a}^{\mu}, \gamma_{\dot{a} a}^{\mu} \psi_{a}^{1}, \bar{\psi}_{\dot{b}}^{2} \gamma_{\dot{b} b}^{\mu}, \gamma_{\dot{b} b}^{\mu} \psi_{b}^{2}, \ldots\right) \\
& U=\sum_{A \in \text { scalars }} \frac{1}{2} m_{A}^{2}\left(\phi^{A}\right)^{2}+\sum_{X \in \text { fermions }} m_{X} \bar{\psi}_{X} \psi_{X} .
\end{aligned}
$$

We will discuss more general examples in Sect. 8 .

We can extract the model functions from the Lagrangian in a constructive manner with the following definitions

$$
\begin{aligned}
{ }_{\alpha} k_{\beta} & =\frac{g_{\mu \nu}}{4} \frac{\vec{\partial}}{\partial\left(\partial_{\mu} \Phi^{\alpha}\right)} \mathrm{L} \frac{\overleftarrow{\partial}}{\partial\left(\partial_{\nu} \Phi^{\beta}\right)} \\
\zeta_{\alpha}^{\mu} & =\frac{2}{i}\left(\mathrm{~L}-\frac{1}{2} g^{\mu \nu} \partial_{\mu} \Phi_{\alpha}^{\alpha} k_{\beta} \partial_{\nu} \Phi^{\beta}\right) \frac{\overleftarrow{\partial}}{\partial\left(\partial_{\mu} \Phi^{\alpha}\right)} \\
, U & =\frac{1}{2} g^{\mu \nu} \partial_{\mu} \Phi_{\alpha}^{\alpha} k_{\beta}(\boldsymbol{\Phi}) \partial_{\nu} \Phi^{\beta}+\frac{i}{2} \zeta_{\alpha}^{\mu}(\boldsymbol{\Phi}) \partial_{\mu} \Phi^{\alpha}-\mathrm{L}
\end{aligned}
$$

Such a construction is important in ensuring the formalism developed in this paper is unique.

\section{Tensors in the field space}

Having defined the field-space supermanifold, we now wish to investigate which field-space tensors can be constructed, given only the Lagrangian of the theory. The ultimate goal is to define a metric for the field space so that we can apply the formalism of the VDW effective action.
The model function $\zeta_{\alpha}^{\mu}$ is a covector in field space, but it is also a spacetime vector. It would be nice to remove the $\mu$ index somehow in order to obtain a pure field-space covector. The simplest way to achieve this would be to contract $\zeta_{\alpha}^{\mu}$ with some spacetime covector. However, there are no spacetime covectors in the theory and $\zeta_{\alpha}^{\mu}$ does not have the right spinor structure to allow a contraction with a $\gamma^{\mu}$ matrix.

Instead, we rely on the observation noted earlier - the spacetime properties of $\zeta_{\alpha}^{\mu}$ are inherited from a $\gamma^{\mu}$ matrix. We can therefore render a pure field-space covector from $\zeta_{\alpha}^{\mu}$ by surgically removing the $\gamma^{\mu}$ matrices.

We can do this in a rigorous way by defining the notion of differentiation with respect to a $\gamma^{\mu}$ matrix. In order to define such a notion, we remember that any matrix $M_{a \dot{a}}$ in spinor space can be uniquely expressed in terms of 16 orthogonal Lorentz-covariant bilinears as

$$
\begin{gathered}
M_{a \dot{a}}=\sum_{i=S, P, V, A, T} a^{(i)} \Gamma_{a \dot{a}}^{(i)}, \\
\Gamma^{(i)} \in\left\{I_{4}, \gamma^{5}, \gamma^{\mu}, \gamma^{\mu} \gamma^{5}, \sigma^{\mu \nu}\right\},
\end{gathered}
$$

where $\Gamma^{(i)}$ (with $i=S, P, V, A, T$ ) are the basis matrices, $a^{(i)}$ is a coefficient, and $\sigma^{\mu \nu} \equiv i / 2\left[\gamma^{\mu}, \gamma^{\nu}\right]$. We can use this expansion to define the general partial differentiation,

$$
\frac{\delta F}{\delta \Gamma^{(i)}} \equiv \lim _{\epsilon^{(i)} \rightarrow 0} \frac{F\left[\Gamma^{(i)} \rightarrow \Gamma^{(i)}+\epsilon^{(i)} I_{4}\right]-F\left[\Gamma^{(i)}\right]}{\epsilon^{(i)}},
$$

such that when applied to a general matrix $M_{a \dot{a}}$ for $\Gamma^{(V)}=$ $\gamma^{\mu}$, we have

$\frac{\delta M_{a \dot{a}}}{\delta \gamma^{\mu}}=a_{\mu}^{(V)} \delta_{a \dot{a}}$.

Note that we must insist that all spinor matrices are written in the form (5.1) before applying (5.2) to ensure that no ambiguities arise from the Clifford algebra.

We may therefore define ${ }^{2}$

$\zeta_{\alpha}=\frac{1}{4} \frac{\delta \zeta_{\alpha}^{\mu}}{\delta \gamma^{\mu}}$.

For the free theory (4.5) this gives

$\zeta_{\alpha}=\left(\mathbf{0}_{N}, \bar{\psi}_{\dot{a}}^{1}, \psi_{a}^{1}, \bar{\psi}_{\dot{b}}^{2}, \psi_{b}^{2}, \ldots\right)$.

The quantity $\zeta_{\alpha}$ defined in (5.4) is a true field-space covector and transforms as

$\zeta_{\alpha} \rightarrow \widetilde{\zeta}_{\alpha}=\zeta_{\beta} \beta\left(J^{-1}\right)_{\alpha}^{\text {sT }}$

under a field redefinition (4.2), where

$\beta\left(J^{-1}\right)_{\alpha}^{s \top}=\Phi^{\beta} \frac{\overleftarrow{\partial}}{\partial \widetilde{\Phi}^{\alpha}}$

2 The factor of $1 / 4$ is included in order to compensate for the factor of 4 arising from the contraction of spacetime indices. 
is the right inverse Jacobian of the transformation.

From $\zeta_{\alpha}$, we can also define a rank-2 tensor

${ }_{\alpha} \lambda_{\beta}=\frac{\vec{\partial}}{\partial \Phi^{\alpha}} \zeta_{\beta}-(-1)^{\alpha+\beta+\alpha \beta} \frac{\vec{\partial}}{\partial \Phi^{\beta}} \zeta_{\alpha}$.

Despite the appearance of partial non-covariant derivatives in (5.8), ${ }_{\alpha} \lambda_{\beta}$ is still a rank-2 tensor and transforms as

${ }_{\alpha} \lambda_{\beta} \rightarrow{ }_{\alpha} \tilde{\lambda}_{\beta}={ }_{\alpha}\left(J^{-1}\right)^{\gamma}{ }_{\gamma} \lambda_{\delta} \delta\left(J^{-1}\right)_{\beta}^{\mathrm{sT}}$.

under a field redefinition (4.2). The reason why ${ }_{\alpha} \lambda_{\beta}$ transforms as a tensor despite the use of partial derivatives is the same reason that the field strength tensor $F_{\mu \nu}$ transforms as a spacetime tensor in QED - namely, any connections added to (5.8) would cancel between the two terms. This cancellation also ensures that ${ }_{\alpha} \lambda_{\beta}$ is left unchanged by the addition of a total derivative to the Lagrangian. Note that the matrix ${ }_{\alpha} \lambda_{\beta}$ is odd under supertransposition (i.e. it is antisupersymmetric) obeying the property $\lambda^{s T}=-\lambda$.

Because of the presence of the scalar fields, the matrix ${ }_{\alpha} \lambda_{\beta}$ is singular even for well behaved theories limiting its usefulness. However, the sum

${ }_{\alpha} \Lambda_{\beta}={ }_{\alpha} k_{\beta}+{ }_{\alpha} \lambda_{\beta}$

is non-singular and therefore has an inverse and a non-zero superdeterminant.

For the free theory (4.5), the definition (5.10) gives the metric

${ }_{\alpha} \Lambda_{\beta}={ }_{\alpha} N_{\beta} \equiv\left(\begin{array}{cccccc}1_{N} & 0 & 0 & 0 & 0 & \ldots \\ 0 & 0 & 1_{4} & 0 & 0 & \ldots \\ 0 & 1_{4} & 0 & 0 & 0 & \ldots \\ 0 & 0 & 0 & 0 & 1_{4} & \ldots \\ 0 & 0 & 0 & 1_{4} & 0 & \ldots \\ \vdots & \vdots & \vdots & \vdots & \vdots & \ddots\end{array}\right)$.

\section{The field-space metric}

We now wish to define a metric for the field-space supermanifold. Such a metric should satisfy the following properties:

1. The metric should be determined solely and uniquely from the action. As a corollary of this property, total derivatives should not contribute to the metric.

2. The metric should transform as a rank 2 field-space tensor.

3. The metric should be supersymmetric, as any antisupersymmetric part will not contribute to the line element of the field-space supermanifold.

4. The metric should not be singular, unless there are nondynamical degrees of freedom.

5. The metric should depend on the fields only and not on their derivatives.
6. The metric for a theory with canonically normalised fields should be given by

$$
{ }_{a} H_{b} \equiv\left(\begin{array}{cccccc}
1_{N} & 0 & 0 & 0 & 0 & \ldots \\
0 & 0 & 1_{4} & 0 & 0 & \ldots \\
0 & -1_{4} & 0 & 0 & 0 & \ldots \\
0 & 0 & 0 & 0 & 1_{4} & \ldots \\
0 & 0 & 0 & -1_{4} & 0 & \ldots \\
\vdots & \vdots & \vdots & \vdots & \vdots & \ddots
\end{array}\right)
$$

which is the analogue of the Euclidean metric that governs locally the supermanifold [48].

The tensor ${ }_{\alpha} \Lambda_{\beta}$ may seem to be a good candidate for the metric, but it does not satisfy property 3 , since $\Lambda^{\mathrm{sT}} \neq \Lambda$. This property is needed for a metric, since only the supersymmetric part of a metric contributes to the field-space line element. The supersymmetric part of ${ }_{\alpha} \Lambda_{\beta}$ is ${ }_{\alpha} k_{\beta}$ which, as we have argued before, cannot be used as a metric because it is singular and so violates property 4 .

One might be tempted to alter the definition (5.8) so that there is a relative plus sign between the two terms instead of a minus. However, as we show in Appendix A, such a definition crucially depends on possible total derivatives that one could add to the Lagrangian and so it violates property 1.

Instead we make use of a useful property of supermanifolds, which is inherited from their relation to ordinary Riemannian manifolds. A supermanifold can always be made locally flat by a suitable change of coordinates [60]. Therefore, by switching to these local inertial coordinates, we can render the field-space metric into a known, simple form.

Mathematically, this is expressed in terms of vielbeins [61] as follows:

${ }_{\alpha} G_{\beta}={ }_{\alpha} e^{a}{ }_{a} H_{b}{ }^{b} e_{\beta}^{\mathrm{sT}}$,

where ${ }_{\alpha} G_{\beta}$ is the field-space metric, ${ }_{\alpha} e^{a}$ are the vielbeins and ${ }_{a} H_{b}$ is defined in (6.1).

But, we know from property 6 that in the local inertial frame the fields should be locally canonical with the same kinetic terms as (4.5). Therefore, in the local inertial frame, we must have ${ }_{a} \Lambda_{b}={ }_{a} N_{b}$ as shown in (5.11) and so

${ }_{\alpha} \Lambda_{\beta}={ }_{\alpha} e^{a}{ }_{a} N_{b}{ }^{b} e_{\beta}^{\mathrm{sT}}$.

The only unknowns in (6.3) are the vielbeins and thus we can use this equation to calculate ${ }_{\alpha} e^{a}$ and, hence, the field-space metric ${ }_{\alpha} G_{\beta}$.

The relation (6.3) does not define the vielbeins ${ }_{\alpha} e^{a}$ uniquely, but only up to a matrix ${ }_{a} X^{b}$ that satisfies

${ }_{a} X^{c}{ }_{c} N_{d}{ }^{d} X_{b}^{\mathrm{ST}}={ }_{a} N_{b}$. 
The most general such matrix can be written as a product of two other matrices as

${ }_{a} X^{b}={ }_{a} Y^{c}{ }_{c} X_{0}^{b}$.

The second matrix ${ }_{c} X_{0}^{b}$ is given by

${ }_{a} X_{0}^{b}=\left(\begin{array}{cccccc}O_{N} & 0 & 0 & 0 & 0 & \ldots \\ 0 & x_{1} & 0 & 0 & 0 & \ldots \\ 0 & 0 & x_{1}^{-1} & 0 & 0 & \ldots \\ 0 & 0 & 0 & x_{2} & 0 & \ldots \\ 0 & 0 & 0 & 0 & x_{2}^{-1} & \ldots \\ \vdots & \vdots & \vdots & \vdots & \vdots & \ddots\end{array}\right)$,

where $O_{N}$ is an orthogonal $N \times N$ matrix and $x_{i}$ (with $i=$ $1,2, \ldots, M)$ are a set of $M$ arbitrary invertible $4 \times 4$ matrices.

The first matrix ${ }_{a} Y^{b}$ in (6.5) accounts for the fact that ${ }_{a} N_{b}$ is invariant under the exchange of $\psi^{I} \leftrightarrow \bar{\psi}^{I}$ for any fermion in the theory. Thus, we can multiply ${ }_{\alpha} e^{a}$ by any matrix that implements such an exchange. There are $2^{M}$ such matrices, which have the form

${ }_{a} Y^{b}=\left(\begin{array}{ccccc}1_{N} & 0 & 0 & \cdots & 0 \\ 0 & y_{1} & 0 & \cdots & 0 \\ 0 & 0 & y_{2} & \cdots & 0 \\ \vdots & \vdots & \vdots & \ddots & \vdots \\ 0 & 0 & 0 & \cdots & y_{M}\end{array}\right)$,

where each of the $y_{i}$ (with $i=1,2, \ldots M$ ) is equal to either

$$
\left(\begin{array}{cc}
1_{4} & 0 \\
0 & 1_{4}
\end{array}\right) \text { or }\left(\begin{array}{cc}
0 & 1_{4} \\
1_{4} & 0
\end{array}\right)
$$

We notice that ${ }_{a} X_{0}^{c}{ }_{c} H_{d}{ }^{d} X_{0 b}^{\mathrm{sT}}={ }_{a} H_{b}$. Consequently, the choice of $O_{N}$ and $x_{i}$ does not affect the field-space metric (6.2). However, ${ }_{a} Y^{c}{ }_{c} H_{d}{ }^{d} Y_{b}^{\text {sT }} \neq{ }_{a} H_{b}$ and hence, the choice of $y_{i}$ will result in $2^{M}$ different possible metrics.

However, only one of these metrics will have a signature compatible with (6.1) with all minus signs on the lower left diagonal. The other $2^{M}-1$ choices of metric will all have at least one minus sign on the upper right diagonal. Therefore, only one choice of ${ }_{a} Y^{b}$ will result in an acceptable field space vielbein. Any other choice would result in a difference between the signature of the local metric and the global metric. Such a signature difference is inadmissible on a supermanifold, in the same way as it is on a regular manifold.

We can, therefore, define a unique field-space metric by insisting that the $\psi^{I} \bar{\psi}^{I}$ components of the metric are positive, while the $\bar{\psi}^{I} \psi^{I}$ components are negative. ${ }^{3}$

\footnotetext{
${ }^{3}$ Note that such a definition can only be made unambiguously if these entries have the same sign everywhere in field-space. This is always the case. For these entries to change sign, they would have to pass through zero, at which point the metric would become singular and the corresponding field would become non-dynamical. Such a situation is unphysical and thus this requirement is unambiguous.
}

With the field-space metric ${ }_{\alpha} G_{\beta}$ determined as described above, we can now proceed to evaluate the field-space connections through the Christoffell symbols

$$
\begin{gathered}
{ }^{\alpha} \Gamma_{\beta \gamma}=\frac{1}{2}{ }^{\alpha} G^{\delta}\left[{ }_{\delta} G_{\beta} \overleftarrow{\partial}_{\gamma}+(-1)^{\beta \gamma}{ }_{\delta} G_{\gamma} \overleftarrow{\partial}_{\beta}\right. \\
\left.-(-1)^{\beta} \vec{\partial}_{\delta \beta} G_{\gamma}\right] .
\end{gathered}
$$

We can then use these connections to define covariant derivatives on the field space

$$
\begin{aligned}
& X^{\alpha} \overleftarrow{\nabla}_{\beta}=X^{\alpha} \frac{\overleftarrow{\partial}}{\partial \Phi^{\beta}}+{ }^{\alpha} \Gamma_{\beta \gamma} X^{\gamma} \\
& X_{\alpha} \overleftarrow{\nabla}_{\beta}=X_{\alpha} \frac{\overleftarrow{\partial}}{\partial \Phi^{\beta}}-X_{\gamma}{ }^{\gamma} \Gamma_{\alpha \beta},
\end{aligned}
$$

with straightforward generalisation to higher order tensors.

The field-space supermanifold can be straightforwardly generalised to an infinite-dimensional configuration-space manifold with coordinates

$\Phi^{\widehat{\alpha}} \equiv \Phi^{\alpha}\left(\boldsymbol{x}_{\alpha}\right)$.

We can define the configuration space metric analogously to (2.7) by

$\widehat{\alpha} G_{\widehat{\beta}}={ }_{\alpha} G_{\beta} \delta^{(4)}\left(\boldsymbol{x}_{\alpha}-\boldsymbol{x}_{\beta}\right)$.

Similarly, we can define the configuration space connections

$$
\begin{aligned}
{ }^{\widehat{\alpha}} \Gamma_{\widehat{\beta} \widehat{\gamma}} & \equiv \frac{1}{2}{ }^{\widehat{\alpha}} G^{\widehat{\delta}}\left[{ }_{\widehat{\delta}} G_{\widehat{\beta}} \frac{\overleftarrow{\delta}}{\delta \phi^{\widehat{\gamma}}}+(-1)^{\beta \gamma}{ }_{\widehat{\delta}} G_{\widehat{\gamma}} \frac{\overleftarrow{\delta}}{\delta \phi^{\widehat{\beta}}}-(-1)^{\beta} \frac{\vec{\delta}}{\delta \phi^{\widehat{\delta}}} \widehat{\widehat{\beta}}_{\widehat{\gamma}}\right] \\
& ={ }^{\alpha} \Gamma_{\beta \gamma} \delta^{(4)}\left(\boldsymbol{x}_{\alpha}-\boldsymbol{x}_{\beta}\right) \delta^{(4)}\left(\boldsymbol{x}_{\alpha}-\boldsymbol{x}_{\gamma}\right)
\end{aligned}
$$

and hence configuration space covariant derivatives

$$
\begin{aligned}
& X^{\widehat{\alpha}} \overleftarrow{\nabla}_{\widehat{\beta}}=X^{\widehat{\alpha}} \frac{\overleftarrow{\delta}}{\delta \phi^{\widehat{\beta}}}+{ }^{\widehat{\alpha}} \Gamma_{\widehat{\beta} \widehat{\gamma}} X^{\widehat{\gamma}}, \\
& X_{\widehat{\alpha}} \overleftarrow{\nabla}_{\widehat{\beta}}=X_{\widehat{\alpha}} \frac{\overleftarrow{\delta}}{\delta \phi^{\widehat{\beta}}}-X_{\widehat{\gamma}} \widehat{\gamma} \Gamma_{\widehat{\beta} \widehat{\alpha}},
\end{aligned}
$$

with straightforward generalisation to higher order tensors.

Finally, we can define a reparametrisation invariant measure

$$
[D \mathrm{M}]=\sqrt{|\operatorname{sdet} G|}\left[D^{N+8 M} \Phi_{q}\right],
$$

where $\boldsymbol{\Phi}_{q}$ represent collectively the quantum field coordinates corresponding to $\boldsymbol{\Phi}$ defined in (6.11). We note that in the local inertial frame $\mid$ sdet $H|=|$ sdet $N \mid$ as can be seen from (6.1) and (5.11). Since these two quantities transform in the same way, we therefore conclude that $|\operatorname{sdet} G|=|\operatorname{sdet} \Lambda|$ in all frames. Thus, we can always replace $G$ with $\Lambda$ in (6.15), without affecting the measure if this proves an easier quantity to calculate. 


\section{The covariant effective action}

With the metric, connections and invariant volume element determined for the field space in the previous section, we are now in a position to make use of the VDW formalism [1,2,13-15] and define the quantum effective action for theories with fermionic degrees of freedom in a reparametrisation-invariant manner. This leads us to the following implicit equation:

$$
\begin{aligned}
\exp (i \Gamma[\boldsymbol{\Phi}])= & \int \sqrt{|\operatorname{sdet} G|}\left[D \boldsymbol{\Phi}_{q}\right] \exp \left(i S\left[\boldsymbol{\Phi}_{q}\right]\right. \\
& \left.-i \int d^{4} x \sqrt{-g} \Gamma[\boldsymbol{\Phi}] \frac{\overleftarrow{\partial}}{\partial \Phi^{\alpha}} \Sigma^{\alpha}\left[\boldsymbol{\Phi}_{q}, \boldsymbol{\Phi}\right]\right)
\end{aligned}
$$

where $\boldsymbol{\Phi}$ denotes the mean field in this section. In the above, $\Sigma^{\alpha}\left[\boldsymbol{\Phi}_{q}, \boldsymbol{\Phi}\right]$ is related to the supergeodesic tangent vector $\sigma^{\alpha}\left[\boldsymbol{\Phi}_{q}, \boldsymbol{\Phi}\right]$ by

$\Sigma^{\alpha}\left[\boldsymbol{\Phi}_{q}, \boldsymbol{\Phi}\right]=\left(C^{-1}\left[\boldsymbol{\Phi}_{q}\right]\right)_{\beta}^{\alpha} \sigma^{\beta}\left[\boldsymbol{\Phi}_{q}, \boldsymbol{\Phi}\right]$,

where $C_{\beta}^{\alpha}\left[\boldsymbol{\Phi}_{q}\right]$ ensures that tadpoles evaluate to zero. In [13], it was found that

$$
\begin{aligned}
C_{\beta}^{\alpha}\left[\boldsymbol{\Phi}_{q}\right] & =\left\langle\sigma^{\alpha}\left[\boldsymbol{\Phi}_{q}, \boldsymbol{\Phi}\right] \overleftarrow{\nabla}_{\beta}\right\rangle \\
& =\left\langle\delta_{\beta}^{\alpha}-(-1)^{\beta \gamma} \frac{1}{3} R_{\gamma \beta \delta}^{\alpha}\left[\boldsymbol{\Phi}_{q}\right] \sigma^{\delta}\left[\boldsymbol{\Phi}_{q}, \boldsymbol{\Phi}\right] \sigma^{\gamma}\left[\boldsymbol{\Phi}_{q}, \boldsymbol{\Phi}\right]+\ldots\right\rangle,
\end{aligned}
$$

where the quantum expectation \langle\rangle is calculated using (7.1). In this VDW formulation, the tangent vector $\sigma^{\alpha}\left[\boldsymbol{\Phi}_{q}, \boldsymbol{\Phi}\right]$ can be expanded to give

$$
\begin{aligned}
-\sigma^{\alpha}\left[\boldsymbol{\Phi}_{q}, \boldsymbol{\Phi}\right]= & -\left(\Phi_{q}^{\alpha}-\Phi^{\alpha}\right) \\
& +\frac{1}{2} \Gamma_{\beta \gamma}^{\alpha}\left[\boldsymbol{\Phi}_{q}\right]\left(\Phi_{q}^{\gamma}-\Phi^{\gamma}\right)\left(\Phi_{q}^{\beta}-\Phi^{\beta}\right)+\cdots .
\end{aligned}
$$

The effective action (7.1) can be expanded covariantly giving at one- and two-loop levels,

$$
\begin{aligned}
\Gamma^{(1)}[\boldsymbol{\Phi}]= & \frac{i}{2} \ln \operatorname{sdet}\left(\vec{\nabla}_{\widehat{\alpha}} S \overleftarrow{\nabla}_{\widehat{\beta}}\right) \\
\Gamma^{(2)}[\boldsymbol{\Phi}]= & \frac{i}{8} S \overleftarrow{\nabla}_{\widehat{\alpha}} \overleftarrow{\nabla}_{\widehat{\beta}} \overleftarrow{\nabla}_{\widehat{\gamma}} \overleftarrow{\nabla}_{\widehat{\delta}} \widehat{\delta} \widehat{\gamma} \Delta^{\widehat{\beta} \widehat{\alpha}} \Delta \\
& +(-1)^{\widehat{\gamma} \widehat{\beta}+\widehat{\epsilon}(\widehat{\delta}+\widehat{\beta})} \frac{i}{12}\left(S \overleftarrow{\nabla}_{\widehat{\epsilon}} \overleftarrow{\nabla}_{\widehat{\gamma}} \overleftarrow{\nabla}_{\widehat{\alpha}}\right) \\
& \times^{\widehat{\alpha}} \Delta^{\widehat{\beta} \widehat{\gamma}} \Delta^{\widehat{\delta} \widehat{\epsilon}} \Delta^{\widehat{\zeta}}\left(\vec{\nabla}_{\widehat{\zeta}} \vec{\nabla}_{\widehat{\delta}} \vec{\nabla}_{\widehat{\beta}} S\right)
\end{aligned}
$$

As expected, these expressions are fully reparametrisation invariant.

The two-loop correction (7.6) to the VDW effective action can also be written as a sum of two covariant Feynman diagrams, i.e.

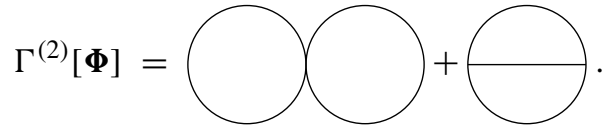

Note that because of the choice (7.3), $\Gamma^{(2)}[\phi]$ contains only 1PI graphs, whereas other possible one-particle reducible diagrams, such as

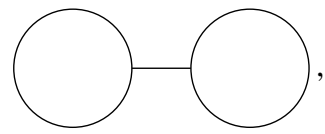

evaluate to zero.

In evaluating the expressions (7.5) and (7.6), we should use the covariant Feynman rules, which are defined by

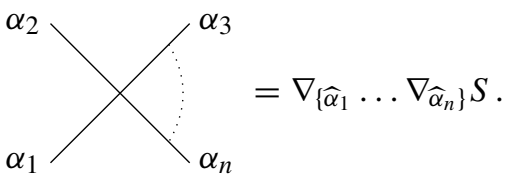

Here the notation $\{\cdots\}$ implies supersymmetrisation over the indices, i.e.

$\left\{\alpha_{i} \cdots \alpha_{n}\right\}=\frac{1}{n !} \sum_{P}(-1)^{P} P\left[\alpha_{i} \cdots \alpha_{n}\right]$,

where $P$ runs over all permutations of the $n$ indices and $(-1)^{P}$ gives -1 when the permutation involves an odd number of fermionic commutations and +1 otherwise.

\section{Examples}

\subsection{Single fermion}

As an explicit example, let us consider a theory with a single scalar field $\phi$ and a single Dirac fermion field $\psi$. The most general Lagrangian for such a theory with up to quadratic kinetic terms is

$$
\begin{aligned}
\mathrm{L}= & \frac{1}{2} k(\phi) \partial_{\mu} \phi \partial^{\mu} \phi-\frac{1}{2} h(\phi) \bar{\psi} \gamma^{\mu} \psi \partial_{\mu} \phi+\frac{i}{2} g(\phi) \bar{\psi} \gamma^{\mu} \partial_{\mu} \psi \\
& -\frac{i}{2} g(\phi) \partial_{\mu} \bar{\psi} \gamma^{\mu} \psi-Y(\phi) \bar{\psi} \psi-V(\phi),
\end{aligned}
$$

where $k, h, g, Y$ and $V$ are arbitrary real functions of $\phi$.

Employing (4.7) and (4.8), we may derive the kinetic model functions for (8.1),

$$
\begin{aligned}
{ }_{\alpha} k_{\beta} & =\left(\begin{array}{ccc}
k(\phi) & 0 & 0 \\
0 & 0 & 0 \\
0 & 0 & 0
\end{array}\right), \\
\zeta_{\alpha}^{\mu} & =\left(i h(\phi) \bar{\psi} \gamma^{\mu} \psi, g(\phi) \bar{\psi} \gamma^{\mu}, g(\phi) \gamma^{\mu} \psi\right) .
\end{aligned}
$$

By means of (5.4), we then obtain

$\zeta_{\alpha}=(i h \bar{\psi} \psi, g \bar{\psi}, g \psi)$ 
and (5.10) yields

${ }_{\alpha} \Lambda_{\beta}=\left(\begin{array}{ccc}k & \frac{1}{2}\left(g^{\prime}-i h\right) \bar{\psi} & \frac{1}{2}\left(g^{\prime}+i h\right) \psi \\ \frac{1}{2}\left(g^{\prime}-i h\right) \bar{\psi} & 0 & g 1_{4} \\ \frac{1}{2}\left(g^{\prime}+i h\right) \psi & g 1_{4} & 0\end{array}\right)$,

where a prime' indicates differentiation with respect to the field $\phi$.

We may now calculate the field-space metric ${ }_{\alpha} G_{\beta}$ through the vielbeins as described in Sect. 6. Solving Eq. (6.3), we see that the vielbeins for this theory are

${ }_{\alpha} e^{a}=\left(\begin{array}{ccc}\sqrt{k} & \frac{g^{\prime}+i h}{2 \sqrt{g}} \psi x & \frac{g^{\prime}-i h}{2 \sqrt{g}} \bar{\psi} x^{-1} \\ 0 & \sqrt{g} x & 0 \\ 0 & 0 & \sqrt{g} x^{-1}\end{array}\right)$,

where $x$ is an arbitrary invertible $4 \times 4$ matrix that can depend on both $\phi$ and $\bar{\psi} \psi{ }^{4}$ As discussed in Sect. 6, the choice of $x(\phi, \bar{\psi} \psi)$ is irrelevant and will cancel out when we calculate the field-space metric.

Using (6.2), we therefore find the field-space metric to be

${ }_{\alpha} G_{\beta}=\left(\begin{array}{ccc}k-\frac{g^{\prime 2}+h^{2}}{2 g} \bar{\psi} \psi & -\frac{1}{2}\left(g^{\prime}-i h\right) \bar{\psi} & \frac{1}{2}\left(g^{\prime}+i h\right) \psi \\ \frac{1}{2}\left(g^{\prime}-i h\right) \bar{\psi} & 0 & g 1_{4} \\ -\frac{1}{2}\left(g^{\prime}+i h\right) \psi & -g 1_{4} & 0\end{array}\right)$.

The superdeterminant of the metric ${ }_{\alpha} G_{\beta}$ to be used in the path integral measure is

$\operatorname{sdet}(G)=\frac{k}{g^{8}}$

Substituting (8.6) into (6.9), we may calculate the fieldspace affine connections of the theory, which we find to be

$$
\begin{aligned}
&{ }^{\phi} \Gamma_{\phi \phi}=\frac{k^{\prime}}{2 k}, \\
& \psi_{a} \Gamma_{\phi \phi}=\left[-\frac{h^{2}+g^{\prime 2}}{4 g^{2}}+\frac{g^{\prime \prime}+i h^{\prime}-\frac{k^{\prime}}{2 k}\left(g^{\prime}+i h\right)}{2 g}\right] \psi_{a}, \\
& \psi_{a} \Gamma_{\psi_{b} \phi}={ }^{\psi_{a}} \Gamma_{\phi \psi_{b}}=\frac{g^{\prime}+i h}{2 g} \delta_{a b}, \\
& \bar{\psi}_{\dot{a}} \Gamma_{\phi \phi}=\left[-\frac{h^{2}+g^{\prime 2}}{4 g^{2}}+\frac{g^{\prime \prime}-i h^{\prime}-\frac{k^{\prime}}{2 k}\left(g^{\prime}-i h\right)}{2 g}\right] \bar{\psi}_{\dot{a}}, \\
& \bar{\psi}_{\dot{b} \Gamma_{\bar{\psi}_{\dot{a}} \phi}}=\bar{\psi}_{\dot{b} \Gamma_{\phi \bar{\psi}_{\dot{a}}}=\frac{g^{\prime}-i h}{2 g} \delta_{\dot{a} \dot{b}},}
\end{aligned}
$$

with all other connections vanishing.

\footnotetext{
${ }^{4}$ Note that there exists another solution

$\alpha e^{a}=\left(\begin{array}{ccc}\sqrt{k} & \frac{g^{\prime}-i h}{2 \sqrt{g}} \bar{\psi} x^{-1} & \frac{g^{\prime}+i h}{2 \sqrt{g}} \psi x \\ 0 & 0 & \sqrt{g} x \\ 0 & \sqrt{g} x^{-1} & 0\end{array}\right)$. But, as discussed in Sect. 6, this
} leads to a metric with the wrong signature.
Knowing the field-space connections in (8.8), we may evaluate the field-space Riemann tensor [48]

$$
\begin{aligned}
{ }^{\alpha} R_{\beta \gamma \delta}= & -{ }^{\alpha} \Gamma_{\beta \gamma} \overleftarrow{\partial}_{\delta}+(-1)^{\gamma \delta \alpha} \Gamma_{\beta \delta} \overleftarrow{\partial}_{\gamma} \\
& +(-1)^{\gamma(\beta+\epsilon) \alpha} \Gamma_{\epsilon \gamma}{ }^{\epsilon} \Gamma_{\beta \delta} \\
& -(-1)^{\delta(\epsilon+\beta+\gamma) \alpha} \Gamma_{\epsilon \delta} \Gamma_{\beta \gamma} .
\end{aligned}
$$

In this way, we find that all the components of ${ }^{\alpha} R_{\beta \gamma \delta}$ vanish identically, which implies that the field space described by (8.6) is flat. Consequently, the theory (8.1) can be made canonical with a suitable field reparametrisation. The reparametrisation in question is

$$
\begin{aligned}
& \phi \rightarrow \widetilde{\phi}=\int_{0}^{\phi} \sqrt{k(\phi)} d \phi, \\
& \psi \rightarrow \widetilde{\psi}=\sqrt{g(\phi)} \exp \left(\frac{i}{2} \int_{0}^{\phi} \frac{h(\phi)}{g(\phi)} d \phi\right) \psi, \\
& \bar{\psi} \rightarrow \widetilde{\bar{\psi}}=\sqrt{g(\phi)} \exp \left(-\frac{i}{2} \int_{0}^{\phi} \frac{h(\phi)}{g(\phi)} d \phi\right) \bar{\psi} .
\end{aligned}
$$

Introducing the field-space multiplet

$\widetilde{\Phi}^{\alpha}=(\widetilde{\phi}, \widetilde{\psi}, \widetilde{\psi})$

we find

$$
\begin{aligned}
\mathrm{L}= & \frac{1}{2} \partial_{\mu} \widetilde{\phi} \partial^{\mu} \widetilde{\phi}+\frac{i \widetilde{\bar{\psi}} \gamma^{\mu} \partial_{\mu} \widetilde{\psi}}{} \\
& -\frac{i}{2} \partial_{\mu} \tilde{\bar{\psi}} \gamma^{\mu} \widetilde{\psi}-\widetilde{Y}(\widetilde{\phi}) \tilde{\bar{\psi}} \widetilde{\psi}-\widetilde{V}(\widetilde{\phi}),
\end{aligned}
$$

is canonical as expected. In (8.12), we have defined

$$
\begin{aligned}
& \tilde{Y}(\tilde{\phi})=g(\phi) Y(\phi), \\
& \tilde{V}(\tilde{\phi})=V(\phi) .
\end{aligned}
$$

Notice that, with the choice

$$
x=\exp \left(\frac{i}{2} \int_{0}^{\phi} \frac{h(\phi)}{g(\phi)} d \phi\right) 1_{4},
$$

the vielbeins in (8.5) can be identified with the Jacobians of the transformation (8.10). Indeed, we see

$$
{ }_{\alpha} e^{a}=\frac{\vec{\partial}}{\partial \Phi^{\alpha}} \widetilde{\Phi}^{a}
$$

As expected, for a flat field space, the vielbeins can be identified with the Jacobian of a transformation and therefore one can move to a field-space frame that is flat everywhere, not just locally.

Let us calculate the one-loop effective potential for (8.12). Since this Lagrangian is canonically normalised, the field space is trivial and hence we can replace covariant derivatives with partial derivatives. We therefore have 


$$
\begin{aligned}
\vec{\nabla}_{\widehat{\alpha}} S & \overleftarrow{\nabla}_{\widehat{\beta}} \\
= & \left(\begin{array}{ccc}
-\square-\widetilde{V}^{\prime \prime}(\widetilde{\phi})-\widetilde{\bar{\psi}} \widetilde{\psi} \widetilde{Y}^{\prime \prime}(\widetilde{\phi}) & -\widetilde{\bar{\psi}} \widetilde{Y}^{\prime}(\widetilde{\phi}) & \widetilde{\psi} \widetilde{Y}^{\prime}(\widetilde{\phi}) \\
\widetilde{\widetilde{\psi}} \widetilde{Y}^{\prime}(\widetilde{\phi}) & 0 & -\not \partial-\widetilde{Y}(\widetilde{\phi}) \\
-\widetilde{\psi} \widetilde{Y}^{\prime}(\widetilde{\phi}) & (\not \partial+\widetilde{Y}(\widetilde{\phi}))^{T} & 0
\end{array}\right) \\
& \times \delta^{(4)}\left(\boldsymbol{x}_{\alpha}-\boldsymbol{x}_{\beta}\right) .
\end{aligned}
$$

Plugging this result into (7.5), we find up to one-loop,

$$
\begin{aligned}
\Gamma[\Phi]= & S[\Phi]-\frac{i}{2} \operatorname{Tr} \ln \left(\square+\widetilde{V}^{\prime \prime}(\widetilde{\phi})\right. \\
& \left.+\widetilde{\bar{\psi}}\left[2\left(\widetilde{Y}^{\prime}(\widetilde{\phi})\right)^{2}(\not \partial+\widetilde{Y})^{-1}-\widetilde{Y}^{\prime \prime}(\widetilde{\phi})\right] \widetilde{\psi}\right) \\
& -i \operatorname{Tr} \ln (\not \partial+\widetilde{Y}(\widetilde{\phi})) .
\end{aligned}
$$

This agrees with previous results in the literature (see eq. (8.49) in [62]).

\subsection{Multiple fermions}

We now generalise the previous example by including $N$ scalars $\phi^{A}$ and $M$ Dirac fermions $\psi^{X}$. The most general Lagrangian derivable from (4.3) for such a theory with up to quadratic kinetic terms is

$$
\begin{aligned}
\mathrm{L}= & \frac{1}{2} g^{\mu v} k_{A B}(\boldsymbol{\Phi}) \partial_{\mu} \phi^{A} \partial_{\nu} \phi^{B}-\frac{1}{2} h_{A X Y}(\boldsymbol{\Phi}) \bar{\psi}^{X} \gamma^{\mu} \psi^{Y} \partial_{\mu} \phi^{A} \\
& +\frac{i}{2} g_{X Y}(\boldsymbol{\Phi})\left(\bar{\psi}^{X} \gamma^{\mu} \partial_{\mu} \psi^{Y}-\partial_{\mu} \bar{\psi}^{X} \gamma^{\mu} \psi^{Y}\right) \\
& +\frac{i}{2} j_{W X Y Z}(\boldsymbol{\Phi}) \bar{\psi}^{W} \gamma^{\mu} \psi^{X}\left(\bar{\psi}^{Y} \partial_{\mu} \psi^{Z}-\partial_{\mu} \bar{\psi}^{Y} \psi^{Z}\right) \\
& -Y_{X Y}(\boldsymbol{\Phi}) \bar{\psi}^{X} \psi^{Y}-V(\boldsymbol{\phi}) .
\end{aligned}
$$

In the above expression, $W, X, Y$ and $Z$ run over the different species of fermion field, so they lie in the interval $1 \leq(W, X, Y, Z) \leq M$.

The model functions for this theory are

$$
\begin{aligned}
{ }_{\alpha} k_{\beta} & =\left(\begin{array}{cc}
k_{A B} & \mathbf{0}_{N \times 8 M} \\
\mathbf{0}_{8 M \times N} & \mathbf{0}_{8 M \times 8 M}
\end{array}\right), \\
\zeta_{\alpha}^{\mu} & =\left(\begin{array}{c}
i h_{A X Y} \bar{\psi}^{X} \gamma^{\mu} \psi^{Y} \\
g_{Y X} \bar{\psi}^{Y} \gamma^{\mu}+j_{W Y X Z} \bar{\psi}^{W} \gamma^{\mu} \psi^{Y} \bar{\psi}^{Z} \\
g_{X Y} \gamma^{\mu} \psi^{Y}+j_{W Y Z X} \bar{\psi}^{W} \gamma^{\mu} \psi^{Y} \psi^{Z}
\end{array}\right), \\
U & =Y_{X Y} \bar{\psi}^{X} \psi^{Y}+V .
\end{aligned}
$$

For illustration, we consider the case when the Lagrangian (8.18) contains no terms higher than quadratic order in the fermions. We therefore set $j_{W X Y Z}=0$ and assume all other model functions to depend only on the scalar fields. In this case, the Lagrangian takes on the simpler form

$$
\begin{aligned}
\mathrm{L}= & \frac{1}{2} g^{\mu v} k_{A B}(\boldsymbol{\phi}) \partial_{\mu} \phi^{A} \partial_{\nu} \phi^{B}-\frac{1}{2} h_{A X Y}(\boldsymbol{\phi}) \bar{\psi}^{X} \gamma^{\mu} \psi^{Y} \partial_{\mu} \phi^{A} \\
& +\frac{i}{2} g_{X Y}(\boldsymbol{\phi})\left(\bar{\psi}^{X} \gamma^{\mu} \partial_{\mu} \psi^{Y}-\partial_{\mu} \bar{\psi}^{X} \gamma^{\mu} \psi^{Y}\right) \\
& -Y_{X Y}(\boldsymbol{\phi}) \bar{\psi}^{X} \psi^{Y}-V(\boldsymbol{\phi}) .
\end{aligned}
$$

From (5.4), we have, for the Lagrangian (8.20),

$\zeta_{\alpha}=\left(i h_{A X Y} \bar{\psi}^{X} \psi^{Y}, g_{Y X} \bar{\psi}^{Y}, g_{X Y} \psi^{Y}\right)$,

which, using (5.10) gives

$$
\begin{aligned}
{ }_{\alpha} \Lambda_{\beta}= & \left(\begin{array}{cc}
k_{A B}-\left(h_{A W Z, B}-h_{B W Z, A}\right) \bar{\psi}^{W} \psi^{Z} \\
\frac{1}{2}\left(g_{Z X, B}-i h_{B Z X}\right) \bar{\psi}^{Z} \\
\frac{1}{2}\left(g_{X Z, B}+i h_{B X Z}\right) \psi^{Z} \\
\frac{1}{2}\left(g_{Z Y, A}-i h_{A Z Y}\right) \bar{\psi}^{Z} & \frac{1}{2}\left(g_{Y Z, A}+i h_{A Y Z}\right) \psi^{Z} \\
0 & g_{Y X} 1_{4} \\
g_{X Y} 1_{4} & 0
\end{array}\right) .
\end{aligned}
$$

We notice that the Lagrangian (8.20) includes a term with a single derivative of a scalar field as did the Lagrangian (8.1). Such a term should be there generically and even appears in free canonically normalised theories if we perform a suitable field redefinition. To make this more explicit, let us consider a field redefinition of the form

$\psi^{X} \rightarrow \widetilde{\psi}^{X}=\left(K(\boldsymbol{\phi})^{-1}\right)_{Y}^{X} \psi^{Y}$.

In the new fields, the Lagrangian (8.20) becomes

$$
\begin{aligned}
\mathrm{L}= & \frac{1}{2} g^{\mu v} k_{A B} \partial_{\mu} \phi^{A} \partial_{\nu} \phi^{B} \\
& -\frac{1}{2}\left[h_{A X Y} K_{W}^{* X} K_{Z}^{Y}\right. \\
& \left.+i g_{X Y}\left(K_{W}^{* X} \frac{\partial K_{Z}^{Y}}{\partial \phi^{A}}-\frac{\partial K_{W}^{* X}}{\partial \phi^{A}} K_{Z}^{Y}\right)\right] \widetilde{\bar{\psi}}^{W} \gamma^{\mu} \widetilde{\psi}^{Z} \partial_{\mu} \phi^{A} \\
& +\frac{i}{2} g_{X Y} K_{W}^{* X} K_{Z}^{Y}\left(\widetilde{\bar{\psi}}^{W} \gamma^{\mu} \partial_{\mu} \widetilde{\psi}^{Z}-\partial_{\mu} \widetilde{\bar{\psi}}^{W} \gamma^{\mu} \widetilde{\psi}^{Z}\right) \\
& -Y_{X Y} K_{W}^{* X} K_{Z}^{Y} \widetilde{\bar{\psi}}^{W} \widetilde{\psi}^{Z}-V .
\end{aligned}
$$

We see that, even if originally the theory was canonical with $h_{A X Y}=0$ and $g_{X Y}=\delta_{X Y}$, a term with a single derivative of the scalar field will appear after the transformation (8.23).

We may attempt to undo this transformation in order to remove $h_{A X Y}$ with an appropriate field redefinition. If we can find a matrix $K_{Y}^{X}(\boldsymbol{\phi})$ that satisfies

$h_{A X Y} K_{W}^{* X} K_{Z}^{Y}=i g_{X Y}\left(\frac{\partial K_{W}^{* X}}{\partial \phi^{A}} K_{Z}^{Y}-K_{W}^{* X} \frac{\partial K_{Z}^{Y}}{\partial \phi^{A}}\right)$, 
then this term can be removed by performing the transformation (8.23). However, we see that unlike in the single-fermion case, (8.25) cannot always be solved in multi-fermion theories, and hence the $h_{A X Y}$-dependent term must be considered consistently.

In order to obtain the field-space vielbeins, and hence the metric, for this theory we need to solve (6.3) with ${ }_{\alpha} \Lambda_{\beta}$ given by (8.22). This is a very challenging equation to solve in general, and we save such a general solution for future work.

However, we can solve this equation for a simple case with only a single bosonic field $\phi$. This allows us to suppress all indices in the bosonic sector. In this case, (8.22) reduces to

$$
\begin{aligned}
& { }_{\alpha} \Lambda_{\beta} \\
& =\left(\begin{array}{ccc}
k & \frac{1}{2}\left(g_{Z Y}^{\prime}-i h_{Z Y}\right) \bar{\psi}^{Z} & \frac{1}{2}\left(g_{Y Z}^{\prime}+i h_{Y Z}\right) \psi^{Z} \\
\frac{1}{2}\left(g_{Z X}^{\prime}-i h_{Z X}\right) \bar{\psi}^{Z} & 0 & g_{Y X} 1_{4} \\
\frac{1}{2}\left(g_{X Z}^{\prime}+i h_{X Z}\right) \psi^{Z} & g_{X Y} 1_{4} & 0
\end{array}\right) .
\end{aligned}
$$

Solving (6.3), we find the the vielbeins for this theory are

$$
\begin{aligned}
& { }_{\alpha} e^{a}= \\
& \left(\begin{array}{ccc}
\sqrt{k} & \frac{1}{2} \sqrt{g_{Y W}^{-1}}\left(g_{Z W}^{\prime}+i h_{Z W}\right) \psi^{Z} & \frac{1}{2} \sqrt{g_{W Y}^{-1}}\left(g_{Z W}^{\prime}-i h_{Z W}\right) \bar{\psi}^{Z} \\
0 & \sqrt{g}_{Y X} & 0 \\
0 & 0 & \sqrt{g} \\
& 0
\end{array}\right) .
\end{aligned}
$$

Here $\sqrt{g_{X Y}}$ is the matrix square root of $g_{X Y}$, satisfying

$\sqrt{g}_{X Z} \sqrt{g}_{Z Y}=g_{X Y}$

and $\sqrt{g}-1$ is its inverse.

Plugging this into (6.2), we find that the metric for this theory is

$$
\begin{aligned}
{ }_{\alpha} G_{\beta}= & \left(\begin{array}{cc}
k-\frac{1}{2}\left(g_{X Z}^{\prime}-i h_{X Z}\right) g_{Z W}^{-1}\left(g_{W Y}^{\prime}+i h_{W Y}\right) \bar{\psi}^{X} \psi^{Y} \\
\frac{1}{2}\left(g_{Z X}^{\prime}-i h_{Z X}\right) \bar{\psi}^{Z} \\
-\frac{1}{2}\left(g_{X Z}^{\prime}+i h_{X Z}\right) \psi^{Z} \\
-\frac{1}{2}\left(g_{Z Y}^{\prime}-i h_{Z Y}\right) \bar{\psi}^{Z} & \frac{1}{2}\left(g_{Y Z}^{\prime}+i h_{Y Z}\right) \psi^{Z} \\
0 & g_{Y X} 1_{4} \\
-g_{X Y} 1_{4} & 0
\end{array}\right) .
\end{aligned}
$$

Hence, the superdeterminant of the metric, which will appear in the path integral measure, is given by

$$
\operatorname{sdet}(G)=\frac{k}{(\operatorname{det} g)^{8}} \text {. }
$$

Notice that $\operatorname{sdet}(G)=\operatorname{sdet}(\Lambda)$ as expected.

From the metric (8.29), we could proceed as before and compute, through (6.9), the field space connections which enter the VDW effective action. However, such a computation becomes inextricably involved and so we choose to present these results in a future publication.

\section{Discussion}

We have constructed a field-space supermanifold for theories with fermionic degrees of freedom. We have shown how to equip the field space with a proper metric that can be calculated from the classical Lagrangian. This was achieved through the use of field-space vielbeins. Finally, we have shown that this field-space metric can be used to write down the quantum effective action for fermionic theories in a way that is frame- and reparametrisation-invariant.

The addition of fermionic degrees of freedom makes the identification of the field-space metric more involved. For purely bosonic field theories, the Lagrangian contains terms with two derivatives and the coefficients of such a term readily transform as a rank-2 tensor. However, fermions only appear with one derivative in the Lagrangian and so there is no analogous rank-2 tensor that can be immediately identified with the metric. Thus, whereas for bosonic theories the metric can be calculated directly from the Lagrangian, for fermionic theories the metric must be found indirectly by solving (6.3) for the field-space vielbeins.

This difference also means that the relation between the field theory and the geometry of the field space is much more hidden for fermionic theories than it is for bosonic theories. For bosonic theories, the kinetic part of the Lagrangian is proportional to the line element of the field space. Consequently, in the absence of potential terms, the equations of motion of a scalar field theory are the geodesic equations of the field space. In addition, any Noether symmetries of the field theory must obey Killing's equation on the field space [63].

With the addition of fermions, this connection is no longer evident. The Lagrangian bears no direct relation to the line element of the field-space supermanifold and as such, the equations of motion of the fields are not directly related to the geodesic equation for this field space. It would be interesting to examine whether the relation between Noether symmetries of field theory and Killing vectors of the field space still holds in fermionic theories, but such investigations lie beyond the scope of the present work.

Nevertheless, the VDW effective action formalism can still be applied and used to define a frame- and reparametrisation-invariant quantum effective action, as stated in (7.1). Hence, it is possible to describe the complete theory with an effective action that is independent of field reparametrisations. This allows us to draw a clear dividing line between the content of a theory (i.e. the physical observations it predicts) and its representation (how we choose to write the theory down).

We have so far considered only scalar-fermion theories with up to quadratic kinetic terms. An interesting generalization would be to consider theories with higher derivatives. 
If the techniques developed in this paper were to be applied directly to such theories they would lead to a Finslerian metric [64], i.e. one that depends on both the fields and their derivatives. Investigating the effects of such a metric would be a worthwhile task, but is beyond the scope of this current paper.

Other possible directions for future work include the study of field-space torsion or non-metricity. We have so far taken the field-space connections to be the Christoffel symbols for the metric and so have excluded such possibilities. However, it may be advantageous to define torsionful or nonmetric field spaces for certain theories. We note that this would only be possible for theories with a curved field space. For theories with a flat field space, it is always possible to define a parametrisation for which the kinetic terms are canonical, at which point all field-space effects should disappear.

The VDW effective action has been applied to scalar theories [1,2,12-18], gauge theories [1,2,7,30-32] and gravity [1,2,11,21-29]. The addition of fermions in this paper completes the geometrisation of QFTs for a wide range of theories. We are now in a position to express any theory that includes scalar fields, gravity, gauge bosons, or fermions with up to quadratic kinetic terms in a frame- and reparametrisation-invariant manner. It should therefore be straightforward to construct a field-space supermanifold, not only for the generic models presented in this paper, but for realistic theories of high energy physics, including the Standard Model.

Acknowledgements The authors would like to thank Sam Brady, Owen Goodwin and Matthew Kellett for useful comments and discussion on the mathematical properties of supermanifolds. KF is supported by the University of Manchester through the President's Doctoral Scholar Award. The work of SK was supported by the ERC grant 669668 NEO-NAT. The work of AP is supported by the Lancaster - Manchester - Sheffield Consortium for Fundamental Physics under STFC research grant ST/L000520/1.

Data Availability Statement This manuscript has no associated data or the data will not be deposited. [Authors' comment: There is no data related to this publication since it is a purely theoretical work.]

Open Access This article is licensed under a Creative Commons Attribution 4.0 International License, which permits use, sharing, adaptation, distribution and reproduction in any medium or format, as long as you give appropriate credit to the original author(s) and the source, provide a link to the Creative Commons licence, and indicate if changes were made. The images or other third party material in this article are included in the article's Creative Commons licence, unless indicated otherwise in a credit line to the material. If material is not included in the article's Creative Commons licence and your intended use is not permitted by statutory regulation or exceeds the permitted use, you will need to obtain permission directly from the copyright holder. To view a copy of this licence, visit http://creativecomm ons.org/licenses/by/4.0/.

Funded by SCOAP ${ }^{3}$.

\section{A Failed attempt: the covariant metric}

In this appendix we consider an alternative definition of the metric

${ }_{\alpha} \widetilde{G}_{\beta}={ }_{\alpha} k_{\beta}+\vec{\nabla}_{\alpha} \zeta_{\beta}+(-1)^{\alpha+\beta+\alpha \beta} \vec{\nabla}_{\beta} \zeta_{\alpha}$,

which is a supersymmetric rank-2 field-space tensor. We show explicitly why such a definition does not work.

Note that connection terms on the RHS of (A.1) do not cancel as they do in (5.8) and thus the metric ${ }_{\alpha} \widetilde{G}_{\beta}$ appears on both sides of this implicit equation. Such an equation is difficult to solve in general, but we can still solve it in certain cases.

We consider solving (A.1) for the example theory of a single scalar field $\phi$ and a single Dirac fermion $\psi$ shown in (8.1). We take as an ansatz

$$
\begin{aligned}
& { }_{\alpha} \widetilde{G}_{\beta} \\
& \quad=\left(\begin{array}{ccc}
H(\phi)+A(\phi) \bar{\psi} \psi & B(\phi) \bar{\psi}+C(\phi) \psi & D(\phi) \bar{\psi}+E(\phi) \psi \\
-B(\phi) \bar{\psi}-C(\phi) \psi & 0 & G(\phi) \\
-D(\phi) \bar{\psi}-E(\phi) \psi & -G(\phi) & 0
\end{array}\right) .
\end{aligned}
$$

This is the most general ansatz compatible with the fermionic structure of the metric. After plugging (A.2) into (A.1) and performing some algebra, we find that the solution is

${ }_{\alpha} \widetilde{G}_{\beta}=\left(\begin{array}{ccc}k(\phi)+A(\phi) \bar{\psi} \psi & B(\phi) \bar{\psi}-B(\phi) \psi \\ -B(\phi) \bar{\psi} & 0 & 0 \\ B(\phi) \psi & 0 & 0\end{array}\right)$,

where $A(\phi)$ and $B(\phi)$ are arbitrary functions of $\phi$.

We can immediately see several problems.

1. The metric (A.3) contains arbitrary functions $A(\phi)$ and $B(\phi)$ and as such, it is not uniquely defined by (A.1) or the Lagrangian (8.1).

2. The metric (A.3) does not reduce to the flat metric in the canonical case with $h=0, g=1$ and $k=1$.

3. The metric (A.3) is singular with $\operatorname{sdet}(\widetilde{G})=\infty$.

4. The metric (A.3) has no dependence on the model functions $h$ and $g$, and so it is disconnected with the fermionic part of the theory that it should describe.

We can find one more problem with the metric (A.1) if we consider adding to the Lagrangian a total derivative term

$\mathrm{L} \rightarrow \widetilde{\mathrm{L}}=\mathrm{L}+\frac{i}{2} \partial_{\mu}\left(t(\phi) \bar{\psi} \gamma^{\mu} \psi\right)$.

Such a boundary term will drop out of the classical action when integrated and thus will not affect the results of the theory. However, if we repeat the above derivation for $\widetilde{\mathrm{L}}$, we 
find the metric defined by (A.1) is

$$
{ }_{\alpha} \widetilde{G}_{\beta}\left(\begin{array}{ccc}
k+A \bar{\psi} \psi & B \bar{\psi}\left[-B+2 \frac{\left(B-i h-t^{\prime}\right) t}{g+t}\right] \psi \\
-B \bar{\psi} & 0 & -2 t \\
{\left[B-2 \frac{\left(B-i h-t^{\prime}\right) t}{g+t}\right] \psi} & 2 t & 0
\end{array}\right),
$$

where $A$ and $B$ are again arbitrary functions of $\phi$. We observe that (A.5) depends strongly on the function $t(\phi)$ even though, as we argued above, this function is irrelevant to the physics of the theory. Note that, in contrast, the tensor ${ }_{\alpha} \Lambda_{\beta}$ defined in (5.10) is not affected by the transformation (A.4) and therefore the metric defined in Sect. 6 does not suffer from this issue.

We conclude from this exercise that (A.1) does not constitute a proper definition of the field-space metric for fermionic theories.

\section{References}

1. G.A. Vilkovisky, The unique effective action in quantum field theory. Nucl. Phys. B 234, 125-137 (1984). https://doi.org/10.1016/ 0550-3213(84)90228-1

2. G.A. Vilkovisky, The Gospel According to DeWitt (1984)

3. F. Sauter, Uber das Verhalten eines Elektrons im homogenen elektrischen Feld nach der relativistischen Theorie Diracs. Z. Phys. 69, 742-764 (1931). https://doi.org/10.1007/BF01339461

4. W. Heisenberg, H. Euler, Consequences of Dirac's theory of positrons. Z. Phys. 98(11-12), 714-732 (1936). https://doi.org/10. 1007/BF01343663 arXiv:physics/0605038

5. V. Weisskopf, The electrodynamics of the vacuum based on the quantum theory of the electron. Kong. Dan. Vid. Sel. Mat. Fys. Med. 14N6, 1-39 (1936)

6. J.S. Schwinger, On gauge invariance and vacuum polarization. Phys. Rev. 82, 664-679 (1951). https://doi.org/10.1103/PhysRev. 82.664

7. G. Kunstatter, Vilkovisky's unique effective action: an introduction and explicit calculation. In: NATO Advanced Research Workshop on Superfield Theories Vancouver, British Columbia, Canada, July 25-August 6, 1986, pp. 503-517 (1986)

8. C.P. Burgess, G. Kunstatter, On the physical interpretation of the Vilkovisky-de Witt effective action. Mod. Phys. Lett. A 2, 875 (1987) [Erratum: Mod. Phys. Lett. A 2, 1003 (1987)]. https://doi. org/10.1142/S0217732387001117

9. G. Kunstatter, Geometrical approach to the effective action. In: Banff Summer Institute: Gravitation, pp. 356-400 (1990)

10. S. Odintsov, The parametrization invariant and gauge invariant effective actions in quantum field theory. Fortsch. Phys. 38, 371391 (1990)

11. K. Finn, S. Karamitsos, A. Pilaftsis, Frame covariance in quantum gravity. Phys. Rev. D 102(4), 045014 (2020). https://doi.org/10. 1103/PhysRevD.102.045014. arXiv:1910.06661 [hep-th]

12. M. Gell-Mann, M. Lévy, The axial vector current in beta decay. Il Nuovo Cimento (1955-1965) 16(4), 705-726 (1960)

13. B.S. DeWitt, The effective action. In: Architecture of Fundamental Interactions at Short Distances: Proceedings, Les Houches 44th
Summer School of Theoretical Physics: Les Houches, France, July 1-August 8, 1985, pt2, pp. 1023-1058 (1987)

14. A. Rebhan, The Vilkovisky-de Witt effective action and its application to Yang-Mills theories. Nucl. Phys. B 288, 832-857 (1987). https://doi.org/10.1016/0550-3213(87)90241-0

15. P. Ellicott, D.J. Toms, On the new effective action in quantum field theory. Nucl. Phys. B 312, 700-714 (1989). https://doi.org/ 10.1016/0550-3213(89)90579-8

16. S. Groot Nibbelink, B.J.W. van Tent, Density perturbations arising from multiple field slow roll inflation (2000). arXiv:hep-ph/0011325

17. S. Groot Nibbelink, B.J.W. van Tent, Scalar perturbations during multiple field slow-roll inflation. Class. Quantum Gravity 19, 613-640 (2002). https://doi.org/10.1088/0264-9381/19/4/302. arXiv:hep-ph/0107272

18. B.J.W. van Tent, Multiple-field inflation and the CMB. Class. Quantum Gravity 21, 349-370 (2004). https://doi.org/10.1088/ 0264-9381/21/2/002. arXiv:astro-ph/0307048

19. D. Burns, S. Karamitsos, A. Pilaftsis, Frame-covariant formulation of inflation in scalar-curvature theories. Nucl. Phys. B907, 785-819 (2016). https://doi.org/10.1016/j.nuclphysb.2016. 04.036. arXiv: 1603.03730

20. S. Karamitsos, A. Pilaftsis, Frame covariant nonminimal multifield inflation. Nucl. Phys. B927, 219-254 (2018). https://doi.org/10. 1016/j.nuclphysb.2017.12.015. arXiv:1706.07011

21. B.S. DeWitt, Quantum theory of gravity. 2. The manifestly covariant theory. Phys. Rev. 162, 1195-1239 (1967). https://doi.org/10. 1103/PhysRev.162.1195

22. B.S. DeWitt, Quantum theory of gravity. 3. Applications of the covariant theory. Phys. Rev. 162, 1239-1256 (1967). https://doi. org/10.1103/PhysRev.162.1239

23. B.S. DeWitt, Quantum theory of gravity. 1. The canonical theory. Phys. Rev. 160, 1113-1148 (1967). https://doi.org/10.1103/ PhysRev.160.1113

24. S.R. Huggins, G. Kunstatter, H.P. Leivo, D.J. Toms, On the unique effective action in five-dimensional Kaluza-Klein theory. Phys. Rev. Lett. 58, 296 (1987). https://doi.org/10.1103/PhysRevLett.58. 296

25. P. Lavrov, S. Odintsov, I. Tyutin, On the unique effective action in field theory. Mod. Phys. Lett. A 3, 1273-1276 (1988). https://doi. org/10.1142/S0217732388001525

26. I. Buchbinder, P. Lavrov, S. Odintsov, Unique effective action in Kaluza-Klein quantum theories and spontaneous compactification. Nucl. Phys. B 308, 191-202 (1988). https://doi.org/10.1016/ 0550-3213(88)90048-X

27. C.F. Steinwachs, Non-minimal Higgs inflation and frame dependence in cosmology. AIP Conf. Proc. 1514(1), 161-164 (2013). https://doi.org/10.1063/1.4791748. arXiv:1301.5543

28. I.G. Moss, Covariant one-loop quantum gravity and Higgs inflation (2014). arXiv:1409.2108

29. M. Bounakis, I.G. Moss, Gravitational corrections to Higgs potentials. JHEP 04, 071 (2018). https://doi.org/10.1007/ JHEP04(2018)071. arXiv: 1710.02987

30. B.S. DeWitt, A gauge invariant effective action. In: Oxford Conference on Quantum Gravity, pp. 449-487 (1980)

31. B.S. DeWitt, The Effective Action (1988)

32. E. Fradkin, A.A. Tseytlin, On the new definition of off-shell effective action. Nucl. Phys. B 234, 509-523 (1984). https://doi.org/10. 1016/0550-3213(84)90075-0

33. A. Helset, A. Martin, M. Trott, The geometric standard model effective field theory. JHEP 03, 163 (2020). https://doi.org/10.1007/ JHEP03(2020)163. arXiv:2001.01453

34. J. Fumagalli, M. Postma, M. Van Den Bout, Matching and running sensitivity in non-renormalizable inflationary models. JHEP 09, 114 (2010). https://doi.org/10.1007/JHEP09(2020)114. arXiv:2005.05905 [hep-ph] 
35. C.N. Yang, R.L. Mills, Conservation of isotopic spin and isotopic gauge invariance. Phys. Rev. 96, 191-195 (1954). https://doi.org/ 10.1103/PhysRev.96.191

36. S.L. Glashow, Partial-symmetries of weak interactions. Nucl. Phys. 22(4), 579-588 (1961). https://doi.org/10.1016/ 0029-5582(61)90469-2

37. S. Weinberg, A model of leptons. Phys. Rev. Lett. 19, 1264-1266 (1967). https://doi.org/10.1103/PhysRevLett.19.1264

38. P.A. Dirac, The quantum theory of the electron. Proc. R. Soc. Lond. A 117, 610-624 (1928). https://doi.org/10.1098/rspa.1928.0023

39. E. Fermi, An attempt of a theory of beta radiation. 1. Z. Phys. 88, 161-177 (1934). https://doi.org/10.1007/BF01351864

40. J. Schwinger, Particles and sources (1969)

41. H. Grassmann, Neue theorie der elektrodynamik. Ann. Phys. 140(1), 1-18 (1845). https://doi.org/10.1002/andp.18451400102

42. F. Berezin, Introduction to Superanalysis (1987)

43. F. Berezin, The Method of Second Quantization (Academic Press, Pure and Applied Physics) (1966)

44. B. Kostant, Graded manifolds, graded lie theory, and prequantization, in Differential Geometrical Methods in Mathematical Physics, ed. by K. Bleuler, A. Reetz (Springer, Berlin, 1977), pp. 177-306

45. M. Batchelor, Supermanifolds, in Group Theoretical Methods in Physics, ed. by W. Beiglböck, A. Böhm, E. Takasugi (Springer, Berlin, 1979), pp. 458-465

46. M. Batchelor, The structure of supermanifolds. Trans. Am. Math. Soc. 253, 329-338 (1979)

47. D.A. Leites, Introduction to the theory of supermanifolds. Russ. Math. Surv. 35(1), 1-64 (1980). https://doi.org/10.1070/ rm1980v035n01abeh001545

48. B. DeWitt, Supermanifolds (Cambridge University Press, Cambridge Monographs on Mathematical Physics, 1992)

49. A. Rogers, Supermanifolds: Theory and applications (2007)

50. D. Volkov, V. Akulov, Is the neutrino a goldstone particle? Phys. Lett. B 46, 109-110 (1973). https://doi.org/10.1016/ 0370-2693(73)90490-5
51. J. Wess, B. Zumino, Supergauge transformations in fourdimensions. Nucl. Phys. B 70, 39-50 (1974). https://doi.org/10. 1016/0550-3213(74)90355-1

52. M. Sohnius, Introducing supersymmetry. Phys. Rep. 128, 39-204 (1985). https://doi.org/10.1016/0370-1573(85)90023-7

53. R.P. Feynman, The theory of positrons. Phys. Rev. 76, 749-759 (1949). https://doi.org/10.1103/PhysRev.76.749

54. A. Salam, J. Strathdee, Super-gauge transformations. Nucl. Phys. B 76(3), 477-482 (1974). https://doi.org/10.1016/ 0550-3213(74)90537-9

55. S. Gates, M.T. Grisaru, M. Rocek, W. Siegel, Superspace or one thousand and one lessons in supersymmetry, Vol. 58 (1983). arXiv:hep-th/0108200

56. Ó. Guajardo, A classical approach to smooth supermanifolds, arXiv e-prints (Oct. 2014). arXiv:1410.7857

57. H. Khudaverdian, T. Voronov, Thick morphisms of supermanifolds, quantum mechanics, and spinor representation. J. Geom. Phys. 148, 103540 (2020). https://doi.org/10.1016/j.geomphys.2019.103540

58. M. Kellett, T.R Morris, Parisi-Sourlas supergravity, arXiv e-prints (2020). arXiv:2005.03400

59. H. Yukawa, On the interaction of elementary particles I. Proc. Phys. Math. Soc. Jpn. 17, 48-57 (1935). https://doi.org/10.1143/PTPS. 1.1

60. B. Riemann, Grundlagen für eine allgemeine theorie der functionen einer veränderlichen complexen grösse, $\mathrm{Ph}$.D. thesis, University of Göttingen (1851)

61. J.S. Schwinger, Quantized gravitational field. Phys. Rev. 130, 1253-1258 (1963). https://doi.org/10.1103/PhysRev.130.1253

62. J. Zinn-Justin, Quantum Field Theory and Critical Phenomena, Vol. 77 (1989)

63. K. Finn, S. Karamitsos, A. Pilaftsis, Eisenhart lift for field theories. Phys. Rev. D 98(1), 016015 (2018). https://doi.org/10.1103/ PhysRevD.98.016015. arXiv:1806.02431

64. P. Finsler, Über Kurven und Flächen in allgemeinen Räumen. Göttingen, Zürich: O. Füssli, 120 S. $8^{\circ}$ (1918) 